\title{
PUVA Photochemotherapy
}

National Cancer Institute

\section{Source}

National Cancer Institute. PUVA Photochemotherapy. NCI Thesaurus. Code C29973.

Phototherapy that combines the use of psoralen with UVA light as a treatment for various skin conditions. 